\title{
Coupled Physarum-Inspired Memristor Oscillators for Neuron-like Operations
}

\author{
Vasileios Ntinas* $^{* \dagger}$, Ioannis Vourkas ${ }^{\ddagger}$, Georgios G. Sirakoulis*, Andrew Adamatzky ${ }^{\S}$ and Antonio Rubio ${ }^{\dagger}$ \\ *Department of Electrical and Computer Engineering \\ Democritus University of Thrace, Xanthi, Greece \\ $\dagger$ Department of Electronic Engineering \\ Universitat Polytécnica de Catalunya, Barcelona, Spain \\ *Department of Electronic Engineering \\ Universidad Técnica Federico Santa María, Valparaíso, Chile \\ $\S$ Unconventional Computing Centre \\ University of the West of England, Bristol, U.K.
}

Email: vntinas@ee.duth.gr, ioannis.vourkas@usm.cl, gsirak@ee.duth.gr, andrew.adamatzky@uwe.ac.uk, antonio.rubio@upc.edu

\begin{abstract}
Unconventional computing has been studied intensively even after the appearance of CMOS technology and currently returned to the spotlight because the latter one is about to reach its physical limits, while the always increasing computational power demand requests for novel unconventional computing solutions. In this area, the oscillatory internal motion mechanism of slime mould, namely Physarum Polycephalum, could serve as an alternative concept for the design and development of electronic circuits that exploit the memristive dynamics and simple LC contours to deliver solutions for computationally hard to be solved problems. In this direction, this work presents how bio-inspired memristive LC oscillators with a coupling capacitor can be synchronized to perform the functionalities of a biological neuron, also able to execute more complex computations, aiming to model biological neural systems much more advanced than the neuron-less slime mould biological organism. This work proposes in a plausible and sufficient manner, a connection path between the function mechanism of a simple biological organism and that of complex biological systems, towards unconventional computation with memristors.
\end{abstract} neuron

Keywords—oscillator, memristor, slime mould, synchronization,

\section{INTRODUCTION}

Acellular slime mould $P$. polycephalum has a sophisticated life cycle [1], which includes fruit bodied, spores, single-cell amoebas, and syncytium. In one phase of its cycle, the slime mould becomes a plasmodium, which is a coenocyte: nuclear divisions occur without cytokinesis. It is a single cell with thousands of nuclei. The plasmodium consumes microscopic particles and bacteria and during its foraging behaviour it spans scattered sources of nutrients with a network of protoplasmic tubes. The plasmodium optimizes its protoplasmic network to span all sources of nutrients, stay away from repellents and minimize transportation of metabolites inside its body. Its ability to optimize its shape [2] attracted attention of biologists, then computer scientists [3] and engineers as well. Thus the field of slime mould computing was born. Details can be found in literature such as the book on Physarum machines [3] and the "bible" of slime mould computing [4]. An extensive range of experimental laboratory prototypes of Physarumbased computing devices has been developed, and few ex- amples include shortest path via annealing and chemotaxis, Towers of Hanoi via shortest path problem, travelling salesman problem, spanning trees, approximation of transport networks, Boolean logical gates, ballistic logical gates, optoelectronic gates, and micro-fluid gates with tactile inputs, all briefly overviewed in [5].

In previous work [6] a question was posed - "Can we make an artificial living neural system from slime mold?", with 'artificial' meaning created on purpose but growing naturally [7]. Such a nervous system could consist of agar blobs colonized by Physarum (which would play the role of neurons) interconnected with each other by protoplasmic tubes (which would play the role of neural terminals). Whilst not being able yet to implement such a prototype of working neural networks based on slime mold, in this work some progress is made towards the design of Physarum-based electronic components, which could form an analogous basis of the future slime mould neural network we aim for. Therefore, the following sections present the first results in this direction, both in the experimental development and in the design and circuitlevel simulation of the corresponding memristor-based building blocks.

\section{PHYSARUM ELECTRONICS AND EXPERIMENTS}

Physiological and biochemical properties of the slime mould $P$. polycephalum allow to use the protoplasmic tubes and the body of the living slime as wires [8], voltage dividers [9], low pass filters [10], oscillators [11], tactile sensors [12], [13] memristors [14], [15], Schottky diodes [15], and transistors [16]. Let us consider two devices relevant to present in this paper, namely oscillators and memristors.

First we refer to a simple experiment conducted for the sake of clarity and completeness of the proposed approach. In particular, a typical setup of Physarum oscillators consists of two planar electrodes ( $1 \mathrm{~cm}$ apart from each other), while 1-2 $\mathrm{ml}$ agar blobs are placed on the electrodes. A Physarum is inoculated on one blob and an oat flake on the other blob (Fig. 1(a)). The Physarum is attracted to the oat flake, grows towards virgin blob and colonizes it. Thus the two electrodes end up connected by a protoplasmic tube. Calcium waves and 


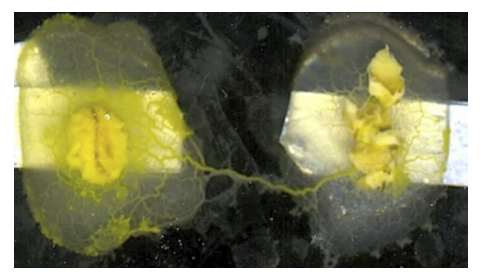

(a)

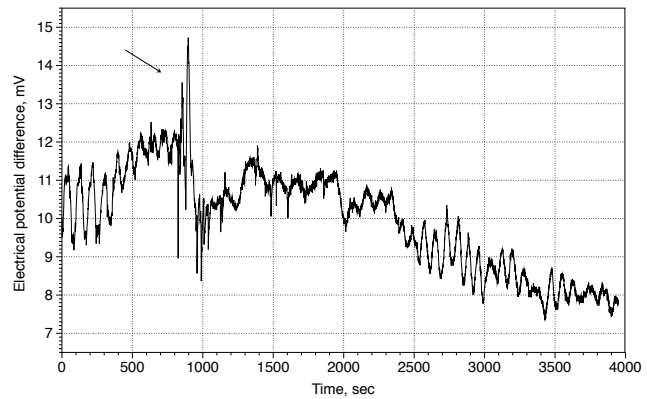

(b)

Fig. 1. Physarum oscillation. (a) Experimental setup: two aluminium electrodes with agar blobs colonized by Physarum are connected by a protoplasmic tube. See [11] for more details. (b) Response of slime mould to trifluoroethane (Sigma Aldrich, UK). Electrical potential difference between two sites of $10 \mathrm{~mm}$ long protoplasmic tube was measured using aluminium electrodes, amplified and digitized with ADC-20 (Pico Technology, UK); $5 \mu \mathrm{L}$ of trifluoroethane applied to a $5 \mathrm{~mm} \times 5 \mathrm{~mm}$ piece of filter paper placed in the Petri dish with slime mould at c. 800 second of recording.

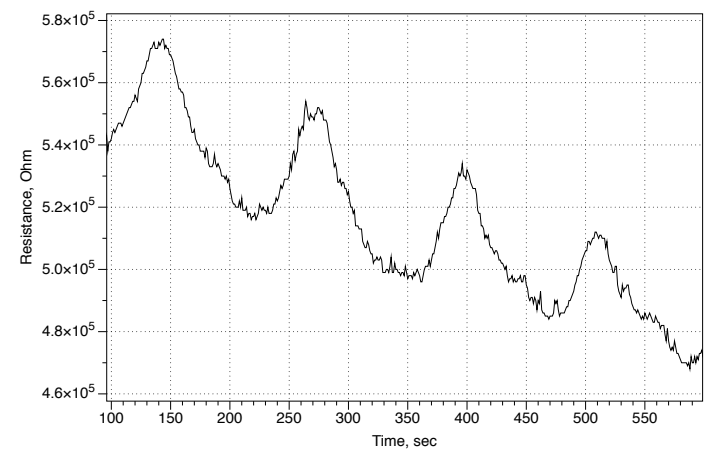

Fig. 2. Dynamics of resistance of the slime protoplasmic tube.

associated electrical charges propagate in the Physarum body and, subsequently, along the protoplasmic tube between the electrodes. Thus oscillations of electrical potential difference between the electrodes are observed as recorded in Fig. 1(b). The oscillations can be interrupted when Physarum is subjected to volatiles of anesthetics, which indicates that propagation of calcium waves and analogs of action potential could be responsible for the oscillations (Fig. 1(b)).

Moreover, propagation of electrical potential is associated with contractions of the protoplasmic tube [17]. Average resistance of a $10 \mathrm{~mm}$ protoplasmic tube was measured $3 \mathrm{MOhm}$. Resistance of the protoplasmic tube exhibits oscillatory behaviour with highly pronounced dominating frequency $0.014 \mathrm{~Hz}$ (Fig. 2). The resistance oscillations have average amplitude $0.59 \mathrm{MOhm}$, minimum amplitude of resistance oscillations observed was $0.11 \mathrm{MOhm}$ and maximum amplitude 1 MOhm. When two Physarum oscillators are electrically

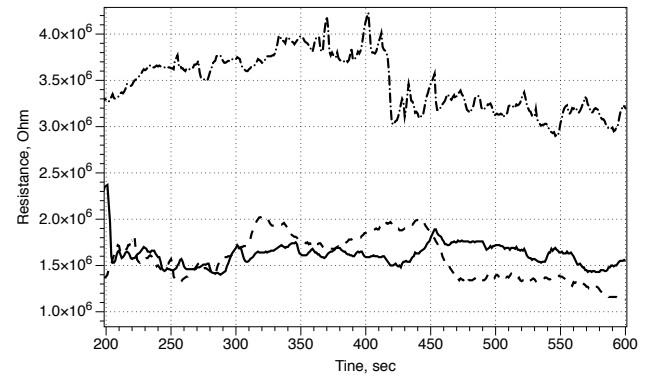

(a)

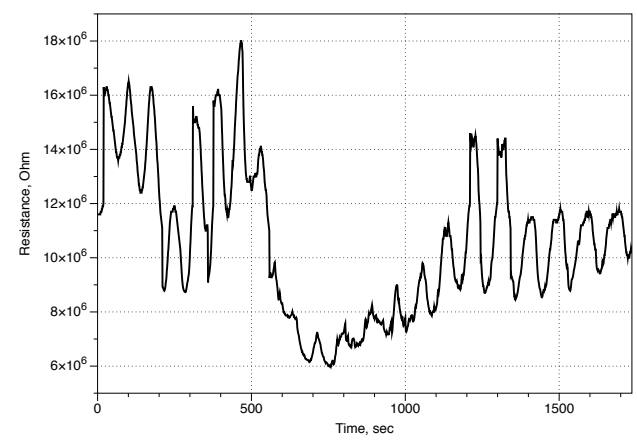

(b)

Fig. 3. Dynamics of resistance of two protoplasmic tubes electrically connected in series. (a) Synchronization of resistance oscillations: solid line and dashed line are single tubes; dash-dot line is for the same two tubes but connected in series. (c) Transient period in oscillatory dynamics, cumulative resistance of two two protoplasmic tubes electrically connected in series.

linked in series, likewise in our aforementioned experiment (Fig. 1(a)) where two electrodes in Petri dishes are connected, the dynamics of their resistance oscillations are synchronized as it can be observed in Fig. 3(a). Sometimes, transient patterns of synchronization can be noticed (Fig. 3a); in fact, in our test the synchronization occurs after the $450^{\text {th }}$ second of the experiment and possible cause of such synchronization could be via electrical potential.

Finally, in regards to the experimental evidence of the possible analogy between Physarum and a memristor, in laboratory experiments of [14] it was demonstrated that protoplasmic tubes of Physarum show current versus voltage profiles consistent with a memristive system. In fact, pronounced hysteresis and memristive effects were exhibited by the slime mould. Thus, the slime mould's protoplasmic tube, being a memristive element, can also act as a low level sequential logic element operated with current spikes, or current transients (see [14]). In such a tentative device, logical input bits are temporarily separated. Memristive properties of the slime mould's protoplasmic tubes gives indications that a range of 'classical' memristor-based neuromorphic architectures could be possibly implemented with Physarum. In particular, considering that memristor is recognized as an analog of a synaptic connection [18], then each protoplasmic tube of Physarum, being a living memristor, may be seen as a synaptic element with memory, whose state is modified depending on its pre-synaptic and postsynaptic activity. Therefore, a network of Physarum's protoplasmic tubes is an associate memory network. A memristor can be also made from Physarum bio-organic electrochemical transistor [15] by removing a drain electrode. 


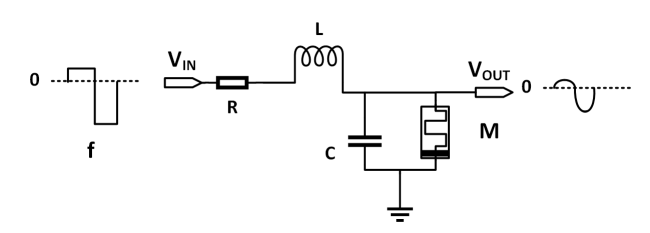

(a)

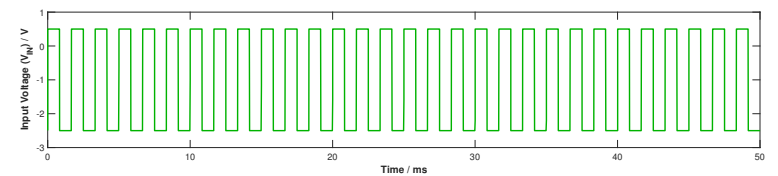

(b)

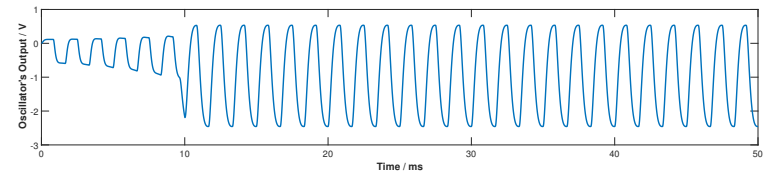

(c)

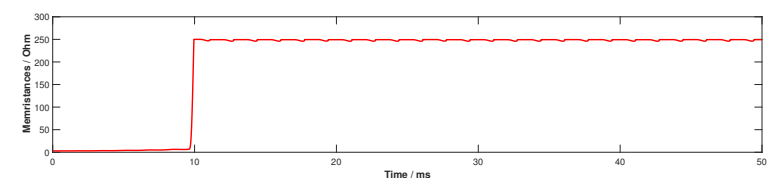

(d)

Fig. 4. (a) Circuit representation of a memristive LC oscillator [19]. (b) Input signal $V_{I N}$. (c) Memristor LC oscillator's output. (d) Memristance evolution in time.

\section{MEMRISTOR LC OSCILlATOR}

As resumed previously, the internal memory abilities and the biological oscillatory system of the slime mould, based on which it is able to recognize periodic changes in the environment, memorize their periods, and adjust its future behavior accordingly [3], [20], can be described by the electronic memristive LC oscillator shown in Fig. 4(a). Such oscillator is based on a classic RLC low-pass filter, where the output response is measured across the capacitor and a memristor, connected in parallel. Including memristor dynamics in the RLC filter adds memory capabilities to the system, thus the output response is controlled also by the time-history of the input signal's characteristics [19], [21], [22], [23].

In this work, a behavioral threshold-based model of a bipolar voltage-controlled memristor with linear ON (low resistive state - LRS) and OFF (high resistive state - HRS) states, was used [24], [25]. Such model is SPICE-compatible and, most importantly, simple-enough to configure in order to capture the basic characteristics of different physical resistive switching devices [26], at a sufficient level of abstraction to enable efficient enough circuit simulations. Hence it was found suitable for the purposes of our application. In specific, the device dynamics are described by the following set of equations:

$$
\begin{aligned}
I_{M}(x, t) & =G(x) \cdot V_{M}(t) \\
M(x) & =\frac{1}{G(x)}=f_{0} \cdot \frac{e^{2 x}}{x}
\end{aligned}
$$

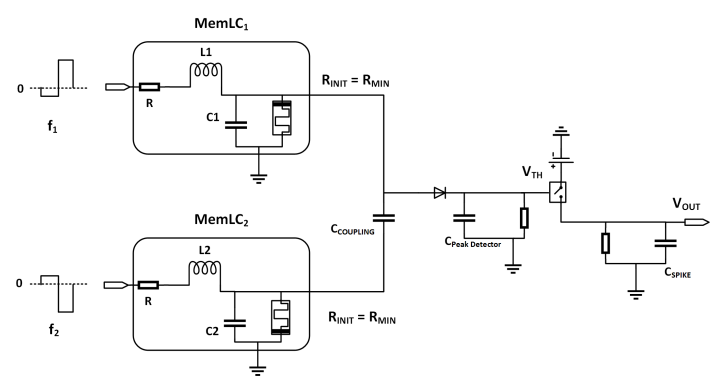

Fig. 5. Coupled memristor LC oscillators and spike generation circuitry.

$$
\begin{array}{ll}
x=x_{0} \cdot\left(1-\frac{m}{r}\right) & \\
\dot{r}= \begin{cases}a_{R E S E T} \cdot \frac{V_{M}+V_{R E S E T}}{c+\left|V_{M}+V_{R E S E T}\right|} & , V_{M}<V_{R E S E T} \\
b \cdot V_{M}, & , V_{R E S E T} \leq V_{M} \leq V_{S E T} \\
a_{S E T} \cdot \frac{V_{M}-V_{S E T}}{c+\left|V_{M}-V_{S E T}\right|} & , V_{M}>V_{S E T}\end{cases}
\end{array}
$$

In such model, resistance switching is attributed to the modulation of an effective tunneling-distance $x$, defined in (3). Eq.(1) and (2) describe the state-dependant Ohm's Law and the state-dependant memristance. Eq. (4) defines the thresholdbased memristance switching rate, being very slow / fast when the applied voltage across the device is below / above the threshold voltage $\left(V_{S E T}\right.$ or $\left.V_{R E S E T}\right)$. Eq. (3) gives a correlation between $r$ (bounded within $\left\{r_{M I N}, r_{M A X}\right\}$ ) and the width of the effective tunneling-distance $x$, which is the state variable. Further information of the physical correspondence of these equations and the role of the parameters can be found in [24].

Regarding the memristor LC oscillator circuit, as long as memristor is initialized in the LRS ( $R_{O N}$ state), any applied voltage of small amplitude $\left(V_{R E S E T}<V_{p}<V_{S E T}\right)$ will not affect much the memristor's state. The LC contour is damped, so the output oscillations decay fast. On the other hand, an on-resonance fixed-amplitude pulse train is able to gradually increase LC contour's output amplitude, and when the latter exceeds the corresponding memristor's threshold $\left(V_{O U T}<\right.$ $\left.V_{R E S E T}\right)$, then the memristor switches to the HRS ( $R_{O F F}$ state). As a result, LC contour is not damped any more and the memristive LC oscillator degenerates to a simple LC oscillator whose resonance frequency is $f_{0}=(2 \pi \sqrt{L C})^{-1}$. A more indepth description of the characteristics of this circuit can be found in [19], where the memristor LC oscillator's response to a wide-range of input signals with varying amplitude and frequency, was studied.

\section{COUpled Memristor LC Oscillators}

Following the biological results of Physarum protoplasmic tubes' emerging synchronization of oscillations and towards the development of a Physarum-based nervous system, the coupling of two memristive LC oscillators to create a neuronlike system was utilized in a novel circuit-level approach, presented here. The proposed coupled system was designed in a completely different manner than any of the previous related works. In fact, the interconnection between the Physarum modules in [27] required a complete set of electronic devices, ie. op-amp and diodes, in order to regulate properly the 


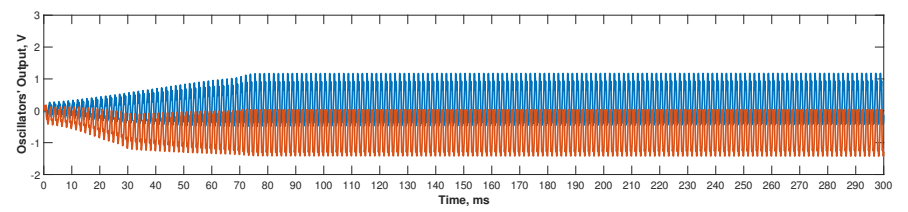

(a)

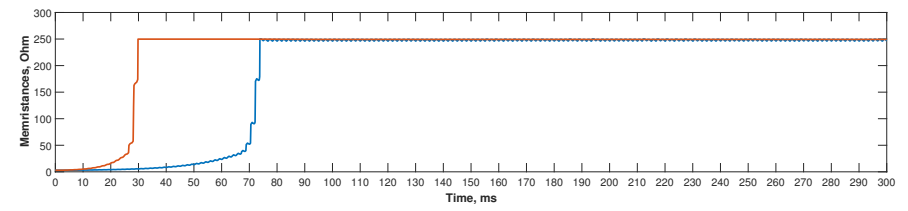

(b)

Fig. 6. (a) Output voltage and (b) memristance evolution in time of $M e m L C_{1}$ (blue) and $M e m L C_{2}$ (orange) for a coupled configuration without any external perturbation.

output signals, while in [28] two sensing systems (input and output control modules) were performing the coupling between Physarum spatial cells.

In this study, in a more simplistic way, the oscillators are coupled via a single capacitor, while, at the same time, they exhibit complementary functionality as their memristor devices are connected with opposite polarization. This can be noted in Fig. 5, where a reverse biased memristor is used in oscillator $M e m L C_{1}$, whereas a forward biased in oscillator $M e m L C_{2}$. The input pulses of each oscillator, which correspond to the food source chemo-attractants in case of Physarum movement, are also working complementary, aiming to the proper functioning of the pulse training process of [19]. Thus, the forward (reverse) biased memristor requires negative (positive) pulses to change its state to high resistance $R_{O F F}$ and consequently to permit full amplitude in-resonance contours. Fig. 6 illustrates the stabilization of both oscillators' output voltage after both memristor's have reached their $R_{O F F}$ state.

However, besides the coupled memristor LC oscillators, Fig. 5 presents in detail the rest of the circuitry required for the neuron-like operation of the proposed system. Particularly, a damping peak detector, consisting of a diode and a parallel $\mathrm{RC}$ circuit, is sensing the voltage on the coupling capacitor's terminal and drives a voltage controlled switch, which is connected with the output RC circuit. During normal operation, the peak detector reaches a maximum value below the switch's threshold due to the coupled oscillators. On the contrary, when an external signal excites one of them, i.e. an incoming spike of another neuron-like coupled system, then the peak detector exceeds the threshold, so the switch closes and the DC source charges the capacitor $C_{O U T}$. As long as $C_{O U T}$ keeps charging, the output signal is used as feedback in both oscillators' inputs with the proper polarity to reset memristors' state to $R_{O N}$. As a result, the voltage of the damping peak detector reduces and when it goes below the switch's threshold, the $C_{O U T}$ is no longer connected to the DC source and starts discharging.

This entire process can be observed in simulation results shown in Fig. 7. After the external excitation, every oscillator's output changes polarity and its memristor gradually switches its state towards $R_{O N}$. Subsequently, $C_{O U T}$ starts discharging and when the system's output voltage is quite low, then every oscillator starts to increase its output amplitude until

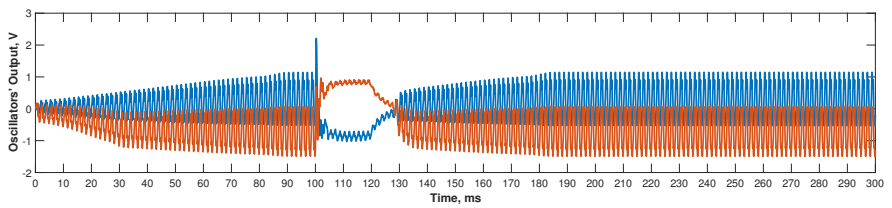

(a)

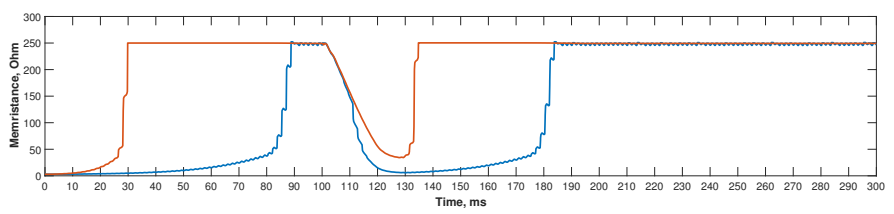

(b)

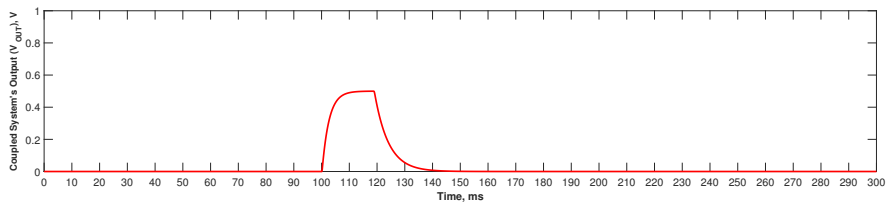

(c)

Fig. 7. (a) Output voltage and (b) memristance evolution in time of $M e m L C_{1}$ (blue) and $M e m L C_{2}$ (orange) for coupled configuration with an external excitation at $M e m L C_{1}$ 's input. (c) Output voltage of the coupled system (Fig. 5).

the coupled system has reached the maximum oscillation state. Therefore, the proposed system depicts the thresholdbased excitation of a real neuron and its refractory time requirements. Moreover, the spike-like required output voltage of the system is achieved as shown in Fig. 7(c), where timing optimization is of course posible by increasing the memristor resistance switching rate parameters, namely $a_{S E T}$ and $a_{R E S E T}$. The chosen frequencies of the simulated example represent just a study case of the coupled system behavior and in the future large scale system the corresponding biologicallybased frequencies will be chosen. In specific, MemLC $C_{1}$ and $M e m L C_{2}$ characteristics are $\left\{R=10 \Omega, L_{1}=1 m H, C_{1}=4 \mu F\right\}$ and $\left\{R=10 \Omega, L_{2}=1 \mathrm{mH}, C_{2}=25 \mu F\right\}$ respectively, whereas memristor device parameters in all the simulated circuits were $f_{0}=0.55$ a.u., $L_{0}=5$ a.u., $m=90$ a.u., $\alpha_{S}=\alpha_{R}=10^{6}$ a.u., $\beta=-10^{4}$ a.u., $c=0.1$ a.u., $\left|V_{S}\right|=\left|V_{R}\right|=1 \mathrm{~V}$, and $\left[R_{O N}, R_{O F F}\right]=$ $[3,250] \Omega$.

\section{Conclusions \& Future Plans}

This work presented the oscillatory phenomena that are observed in Physarum's protoplasmic tubes and proposed a memristive LC oscillator system to be used to create novel neuron-like behavior simply exploiting the emergent synchronization of such phenomena. Further investigation of a suitable neuron-like learning mechanism is in progress and will potentially provide Physarum computing with a completely new area of applications. Some preliminary works in this direction include theoretical designs of oscillatory logical systems [29] and logical gates [30], as well as memory [31] and pattern recognition [32].

\section{ACKNOWLEDGMENT}

This work was supported in part by the Chilean government under the research grants CONICYT FONDECYT Postdoctorado No. 3160042/2016. 


\section{REFERENCES}

[1] S. L. Stephenson, H. Stempen, and I. Hall, Myxomycetes: a handbook of slime molds. Timber Press Portland, Oregon, 1994.

[2] T. Nakagaki, H. Yamada, and A. Toth, "Path finding by tube morphogenesis in an amoeboid organism," Biophysical chemistry, vol. 92, no. 1, pp. 47-52, 2001.

[3] A. Adamatzky, Physarum machines: computers from slime mould. World Scientific, 2010, vol. 74.

[4] A. Adamatzky, Ed., Advances in Physarum machines: Sensing and computing with slime mould. Springer, 2016.

[5] A. Adamatzky, "Thirty seven things to do with live slime mould," in Advances in Unconventional Computing. Springer, 2017, pp. 709-738.

[6] _ "A would-be nervous system made from a slime mold," Artificial life, 2015.

[7] M. A. Bedau, J. S. McCaskill, N. H. Packard, and S. Rasmussen, "Living technology: Exploiting life's principles in technology," Artificial Life, vol. 16, no. 1, pp. 89-97, 2010.

[8] A. Adamatzky, "Physarum wires: Self-growing self-repairing smart wires made from slime mould," Biomedical Engineering Letters, vol. 3, no. 4, pp. 232-241, 2013.

[9] R. Mayne and A. Adamatzky, "Toward hybrid nanostructure-slime mould devices," Nano LIFE, vol. 5, no. 01, p. 1450007, 2015.

[10] J. G. Whiting, B. P. de Lacy Costello, and A. Adamatzky, "Transfer function of protoplasmic tubes of physarum polycephalum," Biosystems, vol. 128 , pp. $48-51,2015$.

[11] A. Adamatzky, "Slime mould electronic oscillators," Microelectronic Engineering, vol. 124, pp. 58-65, 2014

[12] _ - "Slime mould tactile sensor," Sensors and actuators B: chemical, vol. 188 , pp. 38-44, 2013.

[13] _ " "Tactile bristle sensors made with slime mold," Sensors Journal, IEEE, vol. 14, no. 2, pp. 324-332, 2014.

[14] E. Gale, A. Adamatzky, and B. de Lacy Costello, "Slime mould memristors," BioNanoScience, vol. 5, no. 1, pp. 1-8, 2013.

[15] A. Cifarelli, A. Dimonte, T. Berzina, and V. Erokhin, "Non-linear bioelectronic element: Schottky effect and electrochemistry," International Journal of Unconventional Computing, vol. 10, no. 5-6, pp. 375-379, 2014.

[16] G. Tarabella, P. D'Angelo, A. Cifarelli, A. Dimonte, A. Romeo, T. Berzina, V. Erokhin, and S. Iannotta, "A hybrid living/organic electrochemical transistor based on the physarum polycephalum cell endowed with both sensing and memristive properties," Chemical Science, vol. 6, no. 5, pp. 2859-2868, 2015.

[17] T. Sun, S. Tsuda, K.-P. Zauner, and H. Morgan, "Single cell imaging using electrical impedance tomography," in Nano/Micro Engineered and Molecular Systems, 2009. NEMS 2009. 4th IEEE International Conference on. IEEE, 2009, pp. 858-863.

[18] Y. V. Pershin and M. Di Ventra, "Experimental demonstration of associative memory with memristive neural networks," Neural Networks, vol. 23, no. 7, pp. 881-886, 2010

[19] V. Ntinas, I. Vourkas, and G. Sirakoulis, "LC filters with enhanced memristive damping," in IEEE International Symposium on Circuits and Systems (ISCAS) 2015, Aug. 2015, pp. 2664-2667.

[20] T. Saigusa, A. Tero, T. Nakagaki, and Y. Kuramoto, "Amoebae anticipate periodic events," Physical Review Letters, vol. 100, no. 1, p. 018101, 2008.

[21] Y. V. Pershin, S. La Fontaine, and M. Di Ventra, "Memristive model of amoeba learning," Physical Review E, vol. 80, no. 2, p. 021926, 2009.

[22] F. L. Traversa, Y. V. Pershin, and M. Di Ventra, "Memory models of adaptive behavior," IEEE transactions on neural networks and learning systems, vol. 24, no. 9, pp. 1437-1448, 2013.

[23] M. Ziegler, K. Ochs, M. Hansen, and H. Kohlstedt, "An electronic implementation of amoeba anticipation," Applied Physics A, vol. 114, no. 2, pp. 565-570, 2014.

[24] I. Vourkas, A. Batsos, and G. C. Sirakoulis, "Spice modeling of nonlinear memristive behavior," Int. J. of Circuit Theory and Applications, vol. 43, no. 5, pp. 553-565, 2015.
[25] I. Vourkas. (2017) Ijcta spice model netlist. [Online]. Available: https://www.researchgate.net/publication/316656870_Vourkas etal_IJCTA2015_model_SPICE_netlist

[26] G. Sassine, S. La Barbera, N. Najjari, M. Minvielle, C. Dubourdieu, and F. Alibart, "Interfacial versus filamentary resistive switching in $\mathrm{TiO} 2$ and HfO2 devices," Journal of Vacuum Science \& Technology B, vol. 34, no. 1 , p. $012202,2016$.

[27] V. Ntinas, I. Vourkas, G. Sirakoulis, and A. Adamatzky, "Oscillationbased slime mould electronic circuit model for maze-solving computations," IEEE Transactions on Circuits and Systems I: Regular Papers, 2016.

[28] V. Ntinas, I. Vourkas, G. C. Sirakoulis, and A. Adamatzky, "Modeling physarum space exploration using memristors," Journal of Physics D Applied Physics, vol. 50, no. 17, p. 174004, 2017.

[29] A. Adamatzky, "On dynamically non-trivial three-valued logics: oscillatory and bifurcatory species," Chaos, Solitons \& Fractals, vol. 18, no. 5, pp. 917-936, 2003.

[30] J. Borresen and S. Lynch, "Oscillatory threshold logic," PloS one, vol. 7, no. 11, p. e48498, 2012.

[31] V. Calayir and L. Pileggi, "Fully-digital oscillatory associative memories enabled by non-volatile logic," in Neural Networks (IJCNN), The 2013 International Joint Conference on. IEEE, 2013, pp. 1-6.

[32] D. Vodenicarevic, N. Locatelli, J. Grollier, and D. Querlioz, "Synchronization detection in networks of coupled oscillators for pattern recognition," in Neural Networks (IJCNN), 2016 International Joint Conference on. IEEE, 2016, pp. 2015-2022. 